\title{
Image Denoising by Nonlinear Diffusing on Mixed Curvature
}

\author{
Gao Jian ${ }^{\mathrm{a}}$, Zhang Feiyan ${ }^{\mathrm{b}}$, Qin Qianqing ${ }^{\mathrm{c}}$ \\ ${ }^{a}$ LIESMARS, Wuhan University, Wuhan, China \\ ${ }^{b}$ Electronic Information School, Wuhan University, Wuhan, China \\ ${ }^{c}$ LIESMARS, Wuhan University, Wuhan, China
}

\begin{abstract}
A basic problem in the image denoising is noise pressing and edge preserving, while it is difficult to do well in the two aspects at the same time. The Partial Differential Equation (PDE) based methods, such as nonlinear diffusing method, energy minimal method and active contour method, provide a new choice. Here, focus is put on the classic Total Variation and hypersurface minimal problems, which consider regularizing term of isolevel smoothing and mean curvature. In fact, Total Variation smoothing term works well for preserving clear edges and inefficiently in plain areas, while hypersurface minimal smoothing term does well on denoising in plain areas and excessively on edges causing blurring. A projected isolevel curvature is proposed here just as the Beltrami-Laplace operator to mean curvature, considering the gradient while smoothing and keeping edge sharp effectively. And a mixed curvature of mean curvature and projected isolevel curvature forms by a weighting variable. The new denoising method based on the mixed curvature, smoothing in plain areas of image like hypersurface minimal and on edges like a projected isolevel curvature diffusing. Results of relative experiments indicate the proposed mixed curvature denoising method possesses the merits of the two original.
\end{abstract}

Index Terms: Denoise; Mixed Curvature; Total Variation; Hypersurface Minimal

(C) 2012 Published by MECS Publisher. Selection and/or peer review under responsibility of the International Conference on E-Business System and Education Technology

\section{Introduction}

In the past 20 years, the theory of level sets and Partial Differential Equation(PDE) [1] [2] [3] [4] has developed in a variety of directions of image processing, such as denoising and restoration, segmentation, inpainting, decomposing and so on [5] [6]. In the aspect of denoising, Tikhonov and Arsenin gave the Tikhonov model in 1977, then, Rudin, Osher and Fatemi proposed the improved one, Total Variation model [7]. Later, many new methods were proposed with different advantages. Here, focus is put on the hypersurface minimal model and Beltrami flow model. The hypersurface minimal model offers a minimal surface area evolution solution while Beltrami flow driven by a projected mean curvature. But the former brings blurred edges and latter burred. Here provide a mixed solution and the new denoise method provide clear and smooth edges.

Corresponding author:

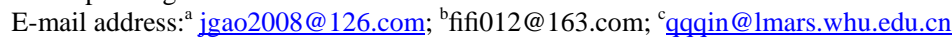




\section{Hypersurface Minimal and Total Variation Smoothing}

Let $\Omega \subset R^{2}$ be bounded and gray image $u: \Omega \rightarrow R$, the hypersurface minimal and Total Variation problems are

$$
\inf _{u} \int_{\Omega} \sqrt{1+|\nabla u|^{2}} d x
$$

and

$$
\inf _{u} \int_{\Omega}|\nabla u| d x
$$

the solution characterized by Euler-Lagrange equation is

$$
\operatorname{div}\left(\frac{\nabla u}{\sqrt{1+|\nabla u|^{2}}}\right)=\kappa_{m}=0
$$

and

$$
\operatorname{div}\left(\frac{\nabla u}{|\nabla u|}\right)=\kappa=0
$$

where, $\kappa_{m}$ is mean curvature of $2 \mathrm{D}$ image surface in $3 \mathrm{D}$ space [8] [9], $\kappa$ is isolevel curvature. The corresponding diffusing equations are

$$
\begin{aligned}
& \frac{\partial u}{\partial t}=\kappa_{m} \\
& \frac{\partial u}{\partial t}=\kappa
\end{aligned}
$$

In fact, displacement of points on surface should be in the curvature directions, namely coordinates of pixels should change during the procedure. The (1) put all the coordinate components on gray value; it makes edges smoothing more than designed. The Total Variation smoothing take place only on the perpendicular direction of gradient, it also makes blurring more in corners than in straight part of edges.

Beltrami flow method make a projection of curvature to gray axis, that is gray change only depend on the corresponding component [10-12]. Beltrami flow diffusing equation is:

$$
\frac{\partial u}{\partial t}=\frac{\kappa_{m}}{\sqrt{1+|\nabla u|^{2}}}=H
$$

$H$ is called Beltrami-Laplace operator. 
Similarly, a "projected" isolevel curvature smoothing equation one provides is like this:

$$
\frac{\partial u}{\partial t}=\frac{\kappa}{\sqrt{1+|\nabla u|^{2}}}=K
$$

$K$ is called "projected curvature" temporarily. A simple example of the four diffusing equations for same time is shown in Fig. 1.
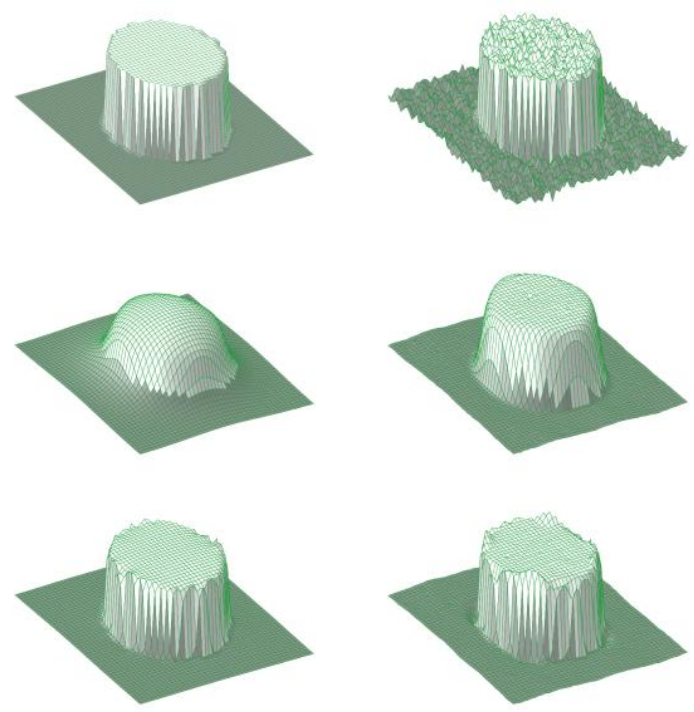

Fig. 1. Evolution of noisy surface

Top: original surface and noisy surface;

middle: hypersurface minimal and Total Variation;

bottom: Beltrami flow and projected isolevel curvature

As shown, Beltrami-Laplace operator and mean curvature all worked better on plain area [13] while isolevel curvature and projected curvature smoothing only on tangent directions of levellines [14] [15]. BeltramiLaplace operator and projected curvature did well on edge better and kept sharper edge than the other two for taking projection into account.

Blurred or burred edge and noisy plain area are bad for processing results, then a compound method designed for a good solution is proposed. Mixing surface curvature and isolevel curvature, non-projected and projected curvature, that is a mixed curvature:

$$
M=M\left(\kappa_{m}, K\right)
$$

Assuming that it removes noises in plain areas effectively and keeps edges smooth and clear. 


\section{How to Get It?}

With the given assumption, firstly a compound function $M(a, b)$ like this:

$$
M(a, b)=(1-\alpha) a+\alpha b \quad \alpha \in(0,1)
$$

$\alpha$ is a weighting variable, it should be decreasing and approach 0 in plain area and 1 on steep edge for the designed goal.

Then the problem is how to distinguish edges from plain areas by the weighting variable. Curvature and gradient all are geometric measure of surface [16]. It is known that the two main curvatures of surface, Gaussian curvature and mean curvature are defined:

$$
\kappa_{g}=\kappa_{1} \cdot \kappa_{2}
$$

where, ${ }^{\kappa_{1}}$ and $\kappa_{2}$ are the principal curvatures, corresponding to the maximum and minimum curvatures. Gaussian curvature takes the product, whose sign is useful for check convex and concave property, mean curvature provides a good measure.

But steep edge doesn't indicate greater curvature than plain areas, a example of one dimensional staircase image on jump point, whose curvature corresponding to different drop heights is shown in Fig. 2.

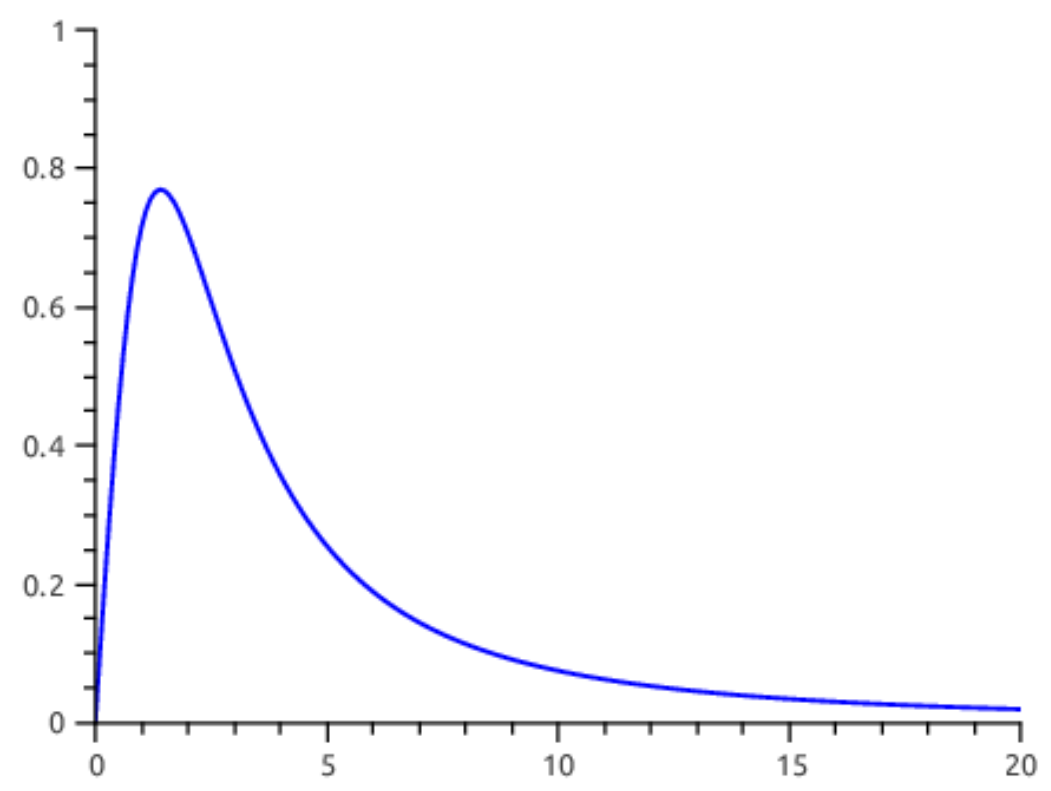

Fig. 2. Curvature on jump point of 1 dimensional staircase image

The steepest staircase edge has no any more difference with plain area on curvature, the same to 2 dimensional image surfaces. However, norm of gradient is a good indicator for gray value varying. 
A version of $\alpha$ provided is like this:

$$
\alpha=\frac{|\nabla u|}{s+|\nabla u|} \quad s \in(0, \infty)
$$

$S$ is a positive constant, controlling the increasing speed of $\alpha$ on norm of gradient, namely the sensitivity to different edges.

The little value of $S$ makes $\alpha$ sensitive to little gray value jump, that is isolevel smoothing determine the final result, then greater $S$ and isolevel smoothing mainly on greater jump. When $S$ approach 0 , the mixed curvature became isolevel curvature, and $\infty$ the Beltrami-Laplace operator. Fig. 3 shows $\alpha$ changing on different $S_{\text {values. }}$

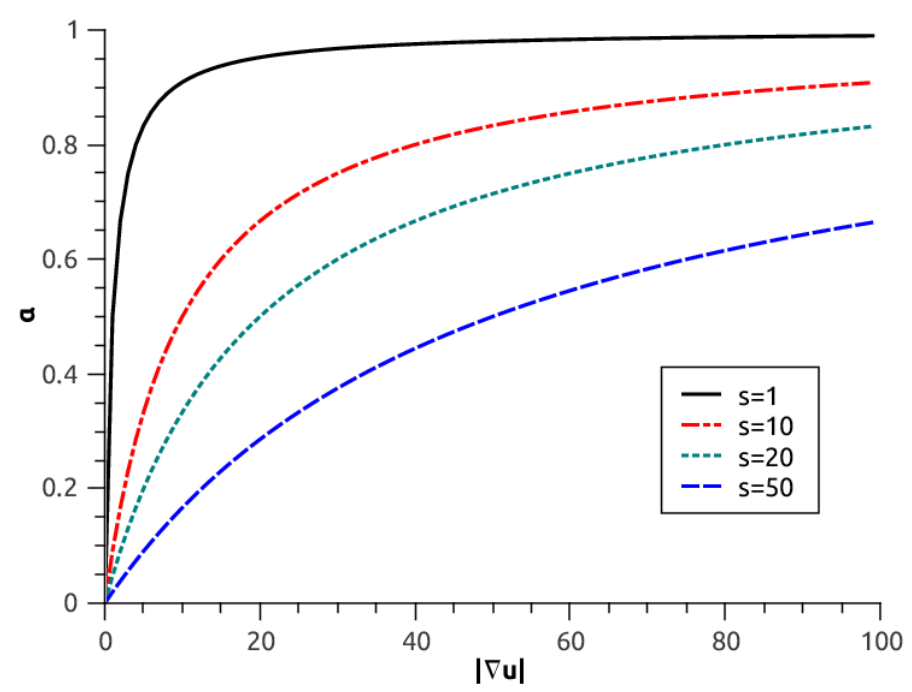

Fig. 3. $\alpha$ with different s.

With the new diffusing equation:

$$
\frac{\partial u}{\partial t}=M\left(\kappa_{m}, K\right)
$$

New evolution results of noisy surface in Fig. 1 are made and shown in Fig. 4.
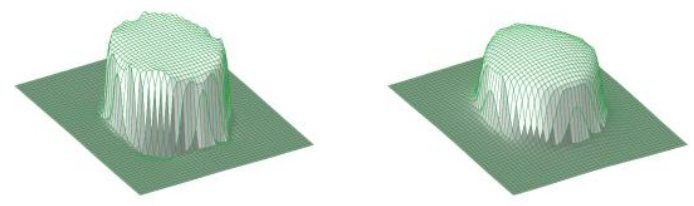

Fig. 4. Evolution with $s=1$ and 5 
The results of $s=1$ and is $s=5$ are better in plain area and edges, little s makes edge sharper and greater s blurring edges. Adding in a fidelity term, a new denoising equation is born from (6)

$$
\frac{\partial u}{\partial t}=f-u+\lambda M\left(\kappa_{m}, K\right)
$$

$$
M\left(\kappa_{m}, K\right)=(1-\alpha) \kappa_{m}+\alpha K
$$

$f$ is the noisy image, $\lambda$ is a positive weighting constant.

\section{Experiments}

The first denoising experiment was carried out on a synthetic image shown in Fig. 5. Adding Gaussian noise of $\sigma=50$ to the original image, then Total Variation, Hypersurface minimal, Beltrami flow, projected isolevel curvature smoothing and the proposed mixed curvature method (7) were applied to it. The results at $\lambda=40$ were shown in Fig. 5.
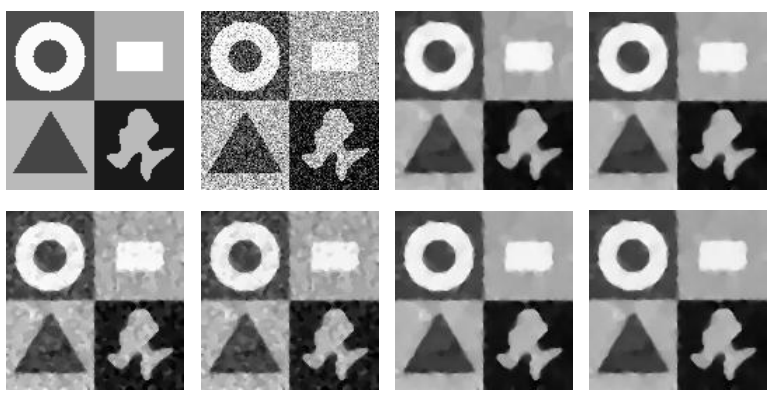

Fig. 5. Denoising of a synthetic image

Top: original, noisy, Total Variation and hypersurface minimal;

bottom: Beltrami flow, projected isolevel curvature and proposed method at $\mathrm{s}=20,50$.

The proposed method got a better result than Beltrami flow and projected isolevel curvature, but seems similar to Total Variation and hypersurface minimal in Fig. 5. The Statistics of Peak Signal Noise Ratio (PSNR) in Tab. I gives a more precise description. The proposed method of mixed curvature at $s=20$ got a higher value than any of others.

Table 1. PSNR of synthetic image experiment results

\begin{tabular}{ccccccc}
\hline Method & Null & TV & HM & Beltrami & Proj. TV & Proposed \\
\hline PSNR/dB & 21.98 & 23.91 & 22.62 & 23.81 & 22.56 & 24.05 \\
\hline
\end{tabular}

The second denoising experiment was carried out on a remote sensing image shown in Fig. 6. After adding Gaussian noise of $\sigma=50$ to the original image, lots of details disappeared. The mixed curvature denoising method was applied to the noisy image with different $\lambda$, the results of $\lambda=70$ and $\lambda=100$ is also shown in Fig. 6. 

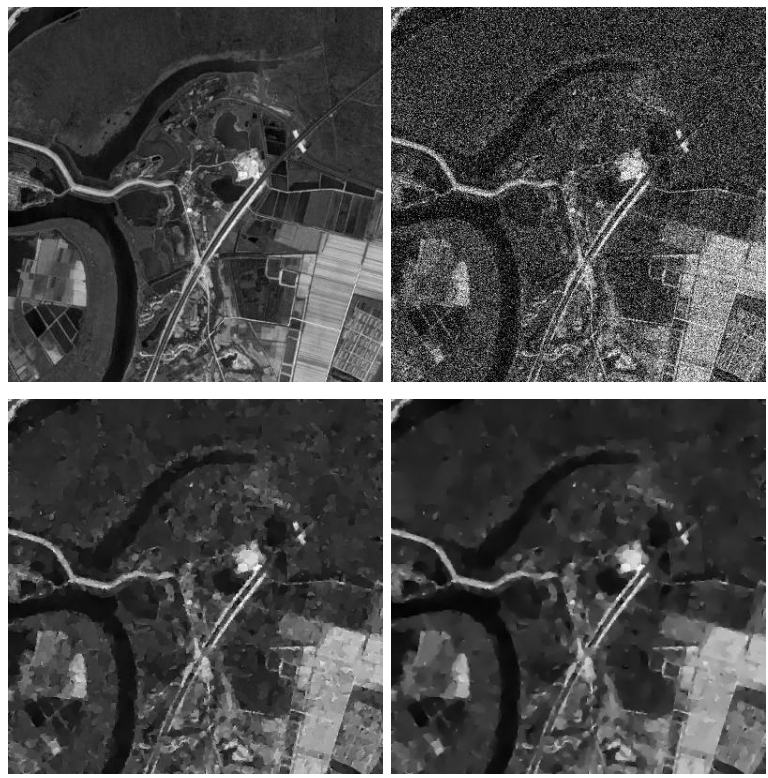

Fig. 6. Denoising of a remote sensing image,

Top: original and noisy image, bottom: proposed method at $\lambda=50,100$.

The processed images kept most all of the useful information and noise was removed obviously, though some details, such as farm balks, couldn't be restored from noisy image for they have been submerged in noise. Restored part of image kept clear edges as same as original mostly. PSNR of them are shown in Fig. 7.

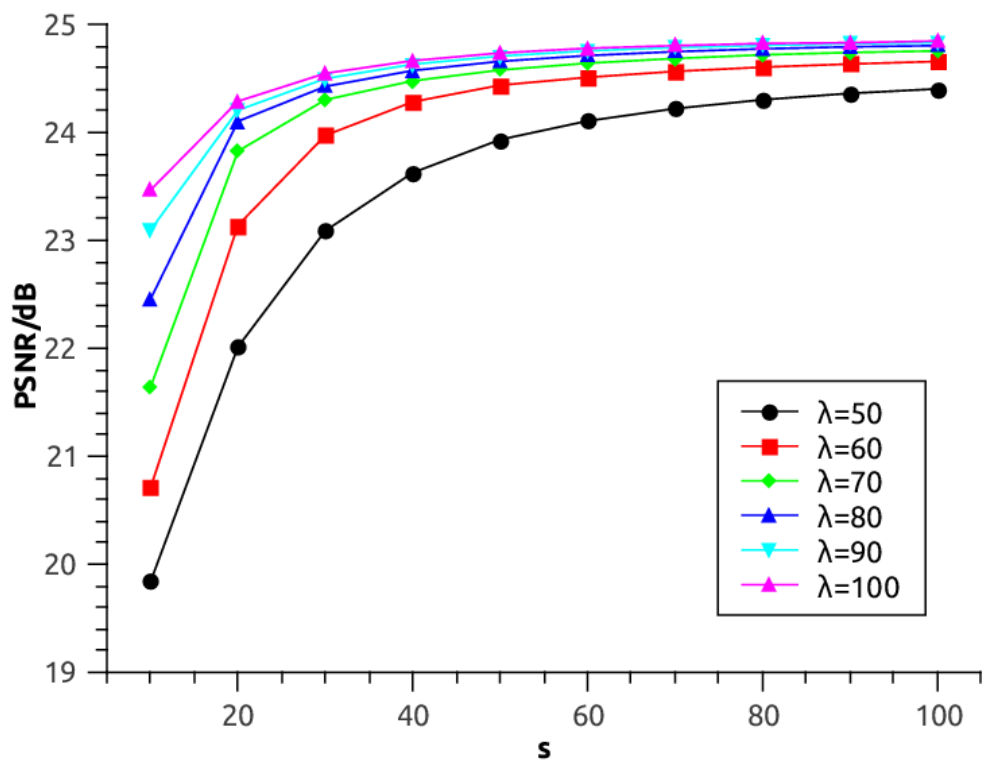

Fig. 7. PSNR of restored remote sensing image 
Values of PSNR ranging from 17.5 to 24.8 , increased with $\lambda$ from 50 to 100 but slower and slower so that there is only little difference between 90 and 100 . At each $\lambda$, PSNR increase with s from 10 to 100 slower and slower, then mostly unchanged when $S$ lager than 80 . So for the noisy remote sensing image, proposed method would get a similar result on PSNR with a $\lambda$ larger than 60 and a s larger than 50 .

\section{Conclusion}

A mixed curvature denoising method is proposed here, which denoise in plain areas of image as hypersurface minimal and smooth at edges on the tangent direction of isolevel and a variable adjust the two status. The special features collect merits of Total Variation and hypersurface minimal, relative experiments carried out and analysis results showed the advantages over other conditional variation denoising method.

The expression of $\alpha$ given here is simple, more smart and powerful function would be found in further research.

\section{References}

[1] M. Elad and M. Aharon, "Image denoising via sparse and redundant representations over learned dictionaries," IEEE Transactions on Image Processing, vol. 15, no. 12, pp. 3736-3745, 2006.

[2] P. Chatterjee and P. Milanfar, "Is denoising dead?" IEEE Transactions on Image Processing, vol. 19, no. 4, pp. 895-911, 2010.

[3] J. A. Sethian, Level Set Methods and Fast Marching Methods. Cambridge University Press, 1999.

[4] S. Osher and R. Fedkiw, Level Set Methods and Dynamic Implicit Surfaces. Springer Press, Feb. 2003.

[5] T. F. Chan and J. Shen, Image Processing and Analysis - Variational, PDE, Wavelet, and Stochastic Methods. Society for Industrial and Applied Mathematics, 2005.

[6] G. Aubert and P. Kornprobst, Mathematical Problems in Image Processing: Partial Differential Equations and the Calculus of Variations. Springer, 2006.

[7] A. I. El-Fallah and G. E. Ford, "On mean curvature diffusion in nonlinear image filtering," Pattern Recognition Letters, vol. 19, no. 5-6, pp. 433-437, 1998.

[8] H. Yu, M. Bennamoun, and C. Chin-Seng, "An extension of min/max flow framework," Image and Vision Computing, vol. 27, no. 4, pp. 342-353, 2009.

[9] N. Sochen, R. Kimmel, and R. Malladi, "A general framework for low level vision," IEEE Transactions on Image Processing, vol. 7, no. 3, pp. 310-318, 1998.

[10] J. A. Sethian, "Image processing via level set curvature flow," Proceedings of the National Academy of Sciences USA, vol. 92, pp. 7046-7050, 1995.

[11] R. Malladi and J. A. Sethian, "Image processing: Flows under $\min / \mathrm{max}$ curvature and mean curvature," Lecture Notes in Computer Science, vol. 1064, pp. 251-262, 1996.

[12] S.-J. Ko and Y.-H. Lee, "Center weighted median filters and their applications to image enhancement," IEEE Transactions on Circuits and Systems, vol. 38, pp. 984-993, 1991.

[13] R. Kimmel, N. A. Sochen, and R. Malladi, "From high energy physics to low level vision. lecture notes in computer science," Lecture Notes In Computer Science, vol. 152, pp. 236-247, 1997.

[14] R. H. Chan, Y. Dong, and M. Hinterm" uller, "An efficient two-phase 11-tv method for restoring blurred images with impulse noise," IEEE Transactions on Image Processing, vol. 19, no. 9, pp. 1731-1739, 2010.

[15] T. Chen and H.Wu, "Adaptive impulse detection using center-weighted median filters," Signal Processing Letters, vol. 8, no. 1, pp. 1-3, 2001.

[16] K. Dabov, V. K. A. Foi, and K. O. Egiazarian, "Image denoising by sparse 3-d transform-domain collaborative filtering,” IEEE Transactions on Image Processing, vol. 16, no. 8, pp. 2080-2095, 2007. 